\title{
BMJ Open Corticosteroids injections versus corticosteroids with hyaluronic acid injections in rhizarthrosis: the randomised multicentre RHIZ'ART trial study protocol
}

\author{
Grégoire Cormier, ${ }^{1}$ Benoit Le Goff, ${ }^{2}$ Amélie Denis, ${ }^{3}$ Stéphane Varin, ${ }^{1}$ \\ Lucie Auzanneau, ${ }^{4}$ Jérôme Dimet, ${ }^{4}$ Aurélie Le Thuaut ${ }^{4}$
}

To cite: Cormier G, Le Goff B, Denis A, et al. Corticosteroids injections versus corticosteroids with hyaluronic acid injections in rhizarthrosis: the randomised multicentre RHIZ'ART trial study protocol. BMJ Open 2019;9:e022553. doi:10.1136/ bmjopen-2018-022553

- Prepublication history for this paper is available online. To view these files, please visit the journal online (http://dx.doi. org/10.1136/bmjopen-2018022553).

Received 23 February 2018 Revised 7 September 2018 Accepted 24 October 2018

Check for updates

(c) Author(s) (or their employer(s)) 2019. Re-use permitted under CC BY-NC. No commercial re-use. See rights and permissions. Published by BMJ.

${ }^{1}$ Service de Rhumatologie, CHD Vendée, La Roche-sur-Yon, France

${ }^{2}$ Service de Rhumatologie, CHU Nantes, Nantes, France

${ }^{3}$ Service de Rhumatologie, $\mathrm{CH}$ Le Mans, Le Mans, Pays de la Loire, France

${ }^{4}$ Methodologist, centre de

Recherche Clinique, CHD

Vendée, La Roche-sur-Yon, Pays de la Loire, France

Correspondence to

Dr Grégoire Cormier;

gregoire.cormier@chd-vendee.fr

\section{ABSTRACT}

Introduction 0steoarthritis of the trapeziometacarpal joint affects approximately $10 \%-25 \%$ of women, especially those who are postmenopausal. It may result in thumb dysfunction. Among the treatments, intra-articular injections of corticosteroid (CS) and hyaluronic acid (HA) are both effective and recommended. However, clinical trials have shown that HA improves functional capacity, whereas CS only produces a decrease in pain. The synergy of these two drugs has not been evaluated. The primary goal of this study was to determine whether the association between HA and CS produce an additional decrease of more pain during thumb movement at three months postinjection, compared to the level of pain relief from CS alone.

Methods and analysis RHIZ'ART is a prospective, multicentre, comparative, randomised, controlled, doubleblind trial. Patients referred to the rheumatology department for thumb rhizarthrosis will receive an injection of betamethasone with $\mathrm{HA}$ or placebo (serum saline) based on central randomisation and stratification by centre. Injections will be given under ultrasound guidance. The primary outcome will compare the pain Visual Analogue Scale with motion at three months for both groups using a mixed model. The expected decrease in pain intensity in the CS group is $25 \%$ and $35 \%$ in the CS with HA group. In order to achieve a $80 \%$ power for detecting this difference with $\alpha$ set at $5 \%$, 73 patients are needed in each group (146 total). The main secondary outcomes are the Cochin score (hand function) and grip strength. Follow-up visits are at 1, 3, 6 and 12 months. Ethics and dissemination The study project has been approved by the appropriate ethics committee (CPP île de France III, 2017-002298-20). In agreement with current French regulations, a signed informed written consent will be obtained from each patient. Results of the main trial and of the secondary endpoints will be submitted for publication in a peer-reviewed journal.

Trial registration number NCT03431584.

\section{INTRODUCTION}

Background and rationale

Rhizarthrosis or trapeziometacarpal (TMC) joint osteoarthritis (OA) is highly

\section{Strengths and limitations of this study}

- This study is a large double-blinded, multicentre, randomised, controlled trial that tests the effect of the association of hyaluronic acid and steroids versus steroids alone in rhizarthrosis treatment.

- The research question relates to a very frequent disease that affects $10 \%-25 \%$ of women.

- The study design contrasts with other ones published in this field that are usually of poor methodological quality.

- Secondary outcomes assess hand function in addition to Visual Analogue Scale pain.

- A non-blinded injecting physician is a limitation of the study design.

prevalent and affects $10 \%-25 \%$ of women, especially those who are postmenopausal. ${ }^{1}$ It affects joints that are unstable and subject to strong pressure. It is often bilateral, but frequently, there is a weak correlation between symptoms and radiological changes. The pain occurs in the 'anatomical snuffbox' and evolves in a fluctuating manner, with no clear correlation between the clinical symptoms and severity of the radiological changes. It may evolve over several years and result in flexion deformity of the base of the thumb into a ' $\mathrm{Z}$ ' form, thus making it dysfunctional.

Treatment modalities consist of non-pharmacological measures (such as resting, immobilisation with splints or braces, ice packs, etc) and pharmacological measures (analgesics, topical or oral non-steroidal anti-inflammatory drugs (NSAIDs), and either corticosteroid (CS) or hyaluronic acid (HA) injections). These injections have been reported to be effective and are commonly used for treatment of OA. The 2007 European League Against Rheumatism recommendations for 
hand OA state that intra-articular injection of longacting CS is effective for painful flares of OA, especially TMC joint $\mathrm{OA}^{\prime}{ }^{2} \mathrm{CS}$ and HA injections have received the marketing authorisation application, but only CSs are currently reimbursed by the French National Health Insurance.

No rigorous or methodological placebo-controlled clinical studies have been carried out to study the effect of these injections. Only Meenagh et al included two small groups of 20 patients enrolled, with minimal injection volumes $(0.25 \mathrm{~mL})$. In view of these methodological issues, they could not demonstrate any differences between the placebo control and CS groups. ${ }^{3}$

In contrast, when comparing CS and HA, they both demonstrated efficacious pain relief and restoration of functional mobility.

In a randomised clinical trial in 2005, Stahl et al compared CS versus HA injections and reported that they both were effective in reducing pain after 1 month, and lasts up to 6 months. However, at 3 months, HA injections were reported to be more effective for improving hand function, and they demonstrated more durable results than CS injections.

Similar results were published in 2006 by Fuchs et a in a randomised study of 56 patients that described pain relief and improved hand function after either type of injection.

More rapid pain relief was obtained with CS (significant relief at 2 weeks) but results were slightly better for HA over time by 26 weeks. Noticeable functional improvement using HA was observed, especially by 6 months.

In 2008, in a clinical trial comparing three injections (CS, HA and placebo), Heyworth et at reported agreement with these observations, without any significant differences among the study and placebo control groups. All three groups (each with 18-22 patients) demonstrated improvement in pain and function; with a more noticeable improvement in the HA group that continue to experience pain relief at 6 months after the injection and also improved function at three and 6 months.

In 2015, Monfort et $a l^{7}$ concluded that 'HA was more effective over time and more efficiently improved functionality and pain in patients with more severe symptoms'.

Concerning the number of injections, a French team led by Roux et al showed that there was no difference in pain relief or improving hand function between groups who received one, two or three HA injections for rhizarthrosis at 1-week intervals.

Both types of injections appear to be efficacious but with different response patterns: CS produced a rapid analgesic and anti-inflammatory action, whereas the HA gave delayed but sustained action over time. It would be worthwhile to investigate the performance of combining HA with CSs for a possibly magnified and synergistic effect. ${ }^{910}$ For knee OA, this combination demonstrated better pain relief than HA alone although knee function did not differ between the two groups. ${ }^{11}$
Some authors have raised the question of safety when combining these two agents, both from clinical and histological aspects. In particular, there are concerns about reduced chondrocyte production and increasing apoptosis that eventually result in cartilage deterioration. However, no increased risks were reported with this combination, neither from clinical ${ }^{12}$ nor histological evidence. $^{13}$

Currently no trials have yet been conducted to evaluate the performance of cortisone combined with HA for treating rhizarthrosis.

Two meta-analyses have been published recently. The first did not find a difference in the use of CS or HA injections for this pathology. ${ }^{14}$ The second stated that 'trials comparing HA with CSs have indicated that HA may be useful in rhizarthrosis-especially for improving functional capacity-and CSs to decrease pain'; the authors concluded that 'the synergy of these two drugs should be investigated further [...] and other comparative trials involving more patients would be useful. ${ }^{15}$ Therefore, our team designed a clinical trial protocol to conduct an evaluation of two groups, one with cortisone and a placebo and the other with concomitant use of HA and cortisone.

\section{Objectives}

Main objective

To compare the results of pain during thumb movement in patients with rhizarthrosis at 3 months post-CS injection with and without HA.

\section{Secondary objectives}

To evaluate whether CS and HA injections result in hand function improvement, reduced pain both at rest and during movement, any recurrences (new injections) and any adverse side effects.

\section{Clinical trial design}

RHIZ'ART is a multicentre, comparative, randomised (in a 1:1 ratio), placebo-controlled, prospective, doubleblind trial with two groups evaluated concurrently. One group will be given betamethasone and placebo injections; while the second experimental group will receive $\mathrm{HA}$ and betamethasone injections.

\section{Methods: participants, interventions and outcomes}

This study will be conducted in accordance with the Standard Protocol Items: Recommendations for Interventional Trials guidelines. ${ }^{16}$

The RHIZ'ART trail is conducting in three rheumatology departments in three hospitals (one medical centre and two general hospitals) in France.

\section{Inclusion criteria}

- Adults $>40$ years old.

- Pain at the thumb base (near the wrist) brought on by direct pressure and by movement. 
- Pain resistant to well-conducted medical therapy, with analgesics, NSAIDs, ice packs with Visual Analogue Scale (VAS) $\geq 4$ for $>3$ months period.

- Radiological observations (Kapandji face +profile) indicative and typical of rhizarthrosis, Eaton and Litter phase II or III, with at least two of the following five radiological elements observed for the TMC joint:

- Marginal osteophytes.

- Joint space pinching.

- Sclerosis of the subchondral space.

- Subchondral geode.

- Absence of osteopaenia

- Patient could understand the protocol and signed the informed consent.

- Patients covered by health insurance.

Non-inclusion criteria

- Known allergy to one of the products (Diprostène or Sinovial mini).

- Analgesic treatment modified within 4 weeks before trial inclusion.

- Patients with symptomatic bilateral rhizarthrosis.

- Scaphoid-trapezial arthrosis.

- Localised or systemic infection.

- Severe and/or uncontrolled hypertension.

- Previous thumb surgery.

- Having inflammatory rheumatism.

- De Quervain tendinopathy, associated with 'trigger finger' pain.

- Previous injections $<6$ months prior to one of the study injections.

- Uncontrolled diabetes.

- Pregnant or lactating women.

- Immunodeficient patients.

- Patients under guardianship, curatorship, not self-sufficient.

- Patients participating in another clinical research trial (except observational research procedures).

- Patients unable to follow the protocol in the investigators' judgement.

\section{Patient and public involvement statement}

Patients were not involved.

\section{Intervention}

After signing the informed consent form for enrolment in the trial, the patient's clinical examinations include various parameters: an initial B-mode ultrasound with Doppler flow measurement, a pain VAS evaluation (both while resting and in motion), the Cochin scale and a grip and the opposition strength in addition to a pain VAS score during the injection procedure.

In order to not bias the clinical evaluation, the physician who clinically evaluates the patient is not the same one who performs the injection. For reproducibility purposes, only 1-2 doctors actually will perform the injections at each centre.
The products being investigated are only injected once on the initial day using two syringes (regardless of the randomised group).

In every medical centre, the injections are performed according to a standardised protocol, provided to the investigators.

The patient is placed in a supine position with his/her arm extended along the body with the hand positioned on a solid surface and the palm facing upward.

The reference point is the longitudinal palmar section of the joint. The TMC joint has been identified as the hypoechoic zone between the two bones.

The joint can be approached perpendicular to the probe, usually lateral to medial, but it could also easily be performed in the other direction depending on the patient's position and the operator's customary habit. In this technique, the needle appears as a hyperechoic point with a comet-tail artefact. The needle should be introduced perpendicular to the probe at a $30^{\circ}-45^{\circ}$ angle until the hyperechoic dot appeared. A subcutaneous needle is used, with local anaesthesia by Xylocaine for the subcutaneous surface and capsule insertion.

The success criterion is lack of resistance during the injection and verification of capsular distension via ultrasound.

This technique was chosen because the intra-articular location can be verified as Di Sante reported when performing intra-articular HA injections perpendicular to the probe. In $100 \%$ of his patients, a convex capsule appearance resulted following the injection, thus indicating the intra-articular site of the product. ${ }^{17}$

According to the randomised group, either $0.5 \mathrm{~mL}$ of $\mathrm{CS}$ and $0.5 \mathrm{~mL}$ of physiological saline will be injected; or $0.5 \mathrm{~mL}$ of $\mathrm{CS}$ and $0.5 \mathrm{~mL}$ of HA.

During the injection, a drape used for the injections is placed vertically on a infusion stand to prohibit the patient from seeing which product is being used.

To ensure homogeneity of the data, all of the clinical evaluations will be performed in a standard manner.

If necessary, each injection was followed by a 48-hour leave of absence from work.

For each patient, a resting brace was worn at night and at rest for 10 days.

Patients were followed for 1-year postinjection for four visits: at $1,3,6$ and 12 months.

\section{Outcomes}

Principle evaluation criterion

The main criterion for evaluating the effectiveness of thumb injections will be pain intensity at the base of the thumb (in the patient's opinion on movement) using a baseline and 3-month postinjection pain VAS for both groups.

This evaluation will be performed by asking the following question: 'how do you evaluate the pain from your hand OA (at rest or during movement)?' in order to standardise the answers. 
The pain intensity of OA at the base of the thumb will be assessed by the patient with scores between 0 and 10 .

This scale has been used as a criterion of therapeutic efficacy in several studies on hand OA. ${ }^{18}$ Its reliability, validity and sensitivity to change in $\mathrm{OA}$ of the hand have been evaluated. Reducing pain at the base of the thumb is one of the major objectives of injections in rhizarthrosis. ${ }^{19}$

\section{Secondary evaluation criteria}

Cochin score at enrolment, and 1, 3, 6and 12 months postinjection. $^{19}$

This score as assessed by the patient incorporates disability measurements as monitored by various daily activities in the kitchen, bathroom, office, getting dressed and others. It varies between 0 and 25; the higher the score, the more the hand function has been affected by the rheumatological pathology. This index has been validated for its reliability, validity and sensitivity to change in hand OA. This questionnaire usually takes only $3 \mathrm{~min}$, with an interobserver correlation of 0.96 and a good construct validity. Another benefit of this scale is the capability to monitor differences between improving and deteriorating patients.

Grip strength and opposition force will be measured using two different JAMAR brand dynamometers according to a standardised methodology ${ }^{20}$;

The first will measure the opposition force in $\mathrm{kg}$. A measurement of the thumb-index finger of the dominant hand and the non-dominant hand will be performed three times. A $1 \mathrm{~min}$ resting period will be observed between each test with the highest value being retained.

The second will measure grip force in $\mathrm{kg}$.

These two strengths will be assessed at enrolment and at 1, 3, 6 and 12 months postinjection.

- Assessing pain VAS with movement on enrolment at 1 , 6 and 12 months postinjection.

- Assessing pain VAS between baseline and 3 months.

This will be done by the patient himself on a weekly basis with pain VAS assessments recorded in a notebook provided to the patient.

- A pain VAS measurement as perceived at the base of the thumb will be noted at rest at baseline, 1, 3, 6 and 12 months postinjection.

- An evaluation of the most intense pain (Worse Pain Score) at baseline, 1, 3, 6 and 12 months postinjection.

- An ultrasound will be performed on enrolment and at 3 months postinjection using the $\mathrm{B}$ and Doppler mode. The ultrasound aspects of the lesions will be graded as stage I, II or III according to the outcome measures in rheumatology principle.

- A pain VAS measurement during the injection will be recorded.

- Verbal scale of the global evolution at 3 months.

The verbal evolution scale is a dynamic criterion for assessing how a disease evolves with respect to a given reference. The use of this type of scale is suggested by the Treaties on Measurements for Research Projects in Osteoarthritis, and the scale has been effective in numerous trials. ${ }^{21}$

During the clinical follow-up visit at 3 months, the patient will be asked the following question: 'Today, in comparison with the beginning of the care (M0), I consider that at the level of my thumb, there is:'

- Worsening.

- Stabilisation or no improvement.

- Modest improvement.

- Average improvement.

- Significant improvement.

- Healing

- The number of CS injections over the 12-month follow-up period.

- Recording the effects and any adverse events over the 12-month follow-up period.

- Noting the consumption of analgesics and NSAIDs (number of days taking analgesics and the accumulated dose from week preceding each follow-up consultation).

- The number of days off work from initially wearing the orthosis over the 12-month follow-up period.

- The possible evolution to a surgical procedure for this OA over the 12 months of follow-up.

\section{Duration of participation}

The participation schedule is described in table 1 .

\section{Sample size}

The study is a prospective trial focusing on pain reduction at 3 months. Pain evaluation is evaluated from the different scores measured at 3 months versus baseline for both groups. According to the literature, we estimated an average score of $6.4 \pm 1.3$ at baseline (Montfort, 2014). ${ }^{7}$

Using this same reference, we assumed a $25 \%$ decrease in pain in the 'with CS alone' group and assumed a $35 \%$ decrease with 'concomitant CS and HA' group.

To prove this difference with $80 \%$ probability and a $5 \%$ alpha risk, the required number of patients is 132 .

To guarantee sufficient probability, an additional 10\% was included in the study, that is, 146 patients in total.

\section{Recruitment}

Participation in the clinical trial will be proposed to any patient who meets the inclusion criteria and is seen or referred to the rheumatology departments in one of the three centres for rhizarthrosis. This recruitment will be carried out in a usual manner, referred by their attending rheumatologist or their treating physician.

\section{METHODS: PROCEDURE ASSIGNMENT Randomisation}

Randomisation will be performed using online software and the patient and evaluators were blinded for both groups. One group of patients will be given HA injections associated with betamethasone and the second group will receive betamethasone and physiological saline 
Table 1 Study timetable

\begin{tabular}{|c|c|c|c|c|c|c|}
\hline Procedure & $\begin{array}{l}1 \text { month } \\
\text { prior to trial }\end{array}$ & $\begin{array}{l}\text { Baseline: } \\
\text { enrolment }\end{array}$ & 1 month & 3 months & 6 months & 12 months \\
\hline $\begin{array}{l}\text { Verification inclusion/non-inclusion } \\
\text { criteria }\end{array}$ & $x$ & & & & & \\
\hline Consent forms signed & & $\mathrm{x}$ & & & & \\
\hline Patient history & $x$ & & & & & \\
\hline Clinical examination & & $x$ & $x$ & $x$ & $x$ & $\mathrm{x}$ \\
\hline Randomisation & & $x$ & & & & \\
\hline $\begin{array}{l}\text { Pain Visual Analogue Scale (VAS) } \\
\text { while resting }\end{array}$ & & $\mathrm{x}$ & $x$ & $x$ & $x$ & $x$ \\
\hline $\begin{array}{l}\text { Pain VAS pinch forefinger-thumb } \\
\text { (movement) }\end{array}$ & & $x$ & $x$ & $x$ & $x$ & $x$ \\
\hline Pain VAS injection & & $x$ & & & & \\
\hline Worse Pain Score & & $x$ & $x$ & $x$ & $x$ & $x$ \\
\hline Cochin Scale & & $x$ & $x$ & $x$ & $x$ & $x$ \\
\hline Global Verbal Evolution Scale & & & & $x$ & & \\
\hline Dynamometer ( $2 \times 3$ measures) & & $x$ & $x$ & $x$ & $x$ & $x$ \\
\hline Ultrasound mode B+Doppler & & $x$ & & $x$ & & \\
\hline Adverse effects & & $x$ & $x$ & $\mathrm{x}$ & $\mathrm{X}$ & $\mathrm{x}$ \\
\hline
\end{tabular}

injections. The randomisation is centre stratified in a 1:1 ratio and done by block randomisation. The randomisation sequence will be generated by a statistician from the Clinical Research Unit.

\section{Blind study}

The product will be concealed from the patient during the injection by placing a vertical screen on the infusion stand in front of the patient. The syringes were labelled and recognisable to the doctor injecting the product, but he is not the same individual as the medical evaluator, who will be blinded with respect to which product will be injected.

\section{METHODS: DATA COLLECTION, MANAGEMENT AND ANALYSIS Data collection}

The data for the trial will be recorded on a web-based electronic case report form (eCRF). The clinical research staff will retrieve the data from each patient's medical record. The data manager, in collaboration with the coordinating investigator, will establish the test database by exporting data from the eCRFs. Any discrepancy in the protocol will be recorded either on the eCRF or on the medical records. Both will be audited by a clinical research assistant in order to ensure that any protocol discrepancies and/or adverse events are noted in the database.

All required information for the protocol will be noted in the electronic logbook. The data will be taken from each patient's medical record (source data) by the participating investigative teams. Any missing data will be coded.

The collected data and medical records will be verified by a clinical research associate to ensure that any discrepancies with the protocol and any adverse events will be fully documented in the database.

The data will be compiled at the end of the study by the data manager and sent to the statistician in charge of the study.

\section{Statistical method}

Statistical analysis will be performed using SAS V.9.4 software. It will incorporate all the required elements of the Consolidated Standards of Reporting Trials statement.

The main analysis is for intention to treat. It will be followed by a per-protocol analysis. No interim analysis is planned.

Patient description is according to the randomisation group at enrolment

The basic characteristics of the overall population and each group will be described, with percentages $(\%)$ for the categorical variables and the minimum, maximum, mean, SD and quartiles for the quantitative variables. No statistical tests will be performed to compare the two groups on enrolment.

\section{Analysis of the main criterion}

The pain VAS measurement with motion at 3 months will be compared for both groups using a mixed model and taking the initial pain VAS into account as a fixed value and the centre as a random value.

Analysis of secondary criteria

Secondary results will be analysed using mixed generalised models (linear or logistic depending on the type of variable). These models will allow us to consider the 
stratification of the randomisation scheme on the centre (considered as random effects).

The change in quantitative variables over time between the two groups (pain, function) will be analysed using models to consider the random effects of interindividual variability in addition to the intraindividual variability for errors in measurement; the effect of the group, the time and their possible interactions will be estimated and tested.

For all statistical tests, $\mathrm{p}<0.05$ will be taken as indications of a significant difference.

\section{METHODS: MONITORING \\ Data monitoring}

Before recruiting any patients, the health professionals from all three medical centres will be trained for the clinical protocol as well as for reporting the data on the CRF. All regulatory documents are available in each centre. The eCRF is a secure, interactive and accessible tool for each centre, provided and managed by the Promotion Department of CHD Vendée (La Roche-sur-Yon, France). In each participating rheumatology unit, physicians and clinical research staff will be in charge of screening patients. The patients will be enrolled by the doctor in accordance with the protocol and data collection rules.

This study follows the 'Reference Methodology' (MR-001) framework for data processing, files and confidentiality as provided by article 54 paragraph 5 of law no $78-17$ dated 6 January 1978 and updated 21 July 2016 by 'Deliberation No. 2016-262'.

The Vendée (Medical Centre) CHD in La Rochesur-Yon, who promoted the study signed their commitment to adhere to this 'Reference Methodology'.

\section{Exceptions}

A patient's family physician could temporarily halt his participation in the clinical trial if there is any suspicion of serious adverse reactions attributable to the product administered during the study. In this event and according to French regulations, a specific procedure is carried out and described in the study protocol.

\section{Audit}

The promotion department of the clinical research unit of CHD Vendée carries out regular audits for collected data (screening, clinical data).

\section{ETHICS AND COMMUNICATION COMMITTEE}

The clinical trial was conducted according to the current version of the Declaration of Helsinki and recommendations of Good Clinical Practice Guidelines.

\section{Informed consent}

In agreement with current French regulations, the doctor obtained a signed informed written consent form from the patient during the appointment to determine eligibility in the month prior to the trial (M-1). If a patient declined to sign the consent, the doctor will treat the patient according to current therapeutic recommendations. A patient should inform the investigator if he decides to quit the study, without incurring any liability or prejudice and thereby not affecting the quality of the care provided. In the event that he withdraws his consent, a computer processing of the personal data already collected would be completed unless otherwise stated in writing beforehand.

\section{Confidentiality}

The trial will be carried out in accordance with the recommendations originating from the French Data Protection Authority. All medical records (source data) recordings will be kept at the investigative centre and the coordinating centre of data management. The electronic database is anonymous and will be stored for 15 years according to Good Clinical Practice guidelines.

\section{Declaration of conflict of interest}

None of the physicians participating in the study have any conflict of interest, financial or otherwise, in connection with the completion of this study. The laboratories MSD France (marketing the DIPROSTENE) and Genevrier (marketing the SYNOVIAL MINI) will not at any time be involved in the development of the design of the protocol and will not interfere in its progress (data collection, analysis and interpretations, publication decision).

\section{Data access}

Access to the final data for analysis will only be made available to the study coordinator and to the Vendée CHD Promotion Department. The investigating physicians have signed contracts to prohibit their access to this information.

\section{Communication}

The study coordinator agrees to communicate the results to any requesting investigators and participants. The results will only be released at the end of the study (either after the last patient's visit or earlier if required). Results of this study will be disseminated by publications, oral communications or posters at medical conferences.

Contributors BLG, GC, LA and AD wrote the manuscript. GC, JD, SV and BLG participated in the study design. ALT wrote the statistical analysis plan and estimated the sample size. GC, JD and LA obtained the grant for the study. All authors contributed to collecting data, critically evaluating the manuscript and have read and approved the final version.

Funding This trial was supported solely by a grant from the Vendee CHD, via an in-house request.

Competing interests None declared.

Patient consent for publication Obtained.

Ethics approval The project was approved by an Ethics Committee (CPP Ile de France III) in Paris, France, on 19 September 2017 (registration number 2017-002298-20).

Provenance and peer review Not commissioned; externally peer reviewed.

Open access This is an open access article distributed in accordance with the Creative Commons Attribution Non Commercial (CC BY-NC 4.0) license, which permits others to distribute, remix, adapt, build upon this work non-commercially, 
and license their derivative works on different terms, provided the original work is properly cited, appropriate credit is given, any changes made indicated, and the use is non-commercial. See: http://creativecommons.org/licenses/by-nc/4.0/.

\section{REFERENCES}

1. Armstrong AL, Hunter JB, Davis TR, et al. The prevalence of degenerative arthritis of the base of the thumb in post-menopausal women. J Hand Surg Br 1994;19:340-1.

2. Zhang W, Doherty M, Leeb BF, et al. EULAR evidence based recommendations for the management of hand osteoarthritis: report of a Task Force of the EULAR Standing Committee for International Clinical Studies Including Therapeutics (ESCISIT). Ann Rheum Dis 2007;66:377-88.

3. Meenagh GK, Patton J, Kynes C, et al. A randomised controlled trial of intra-articular corticosteroid injection of the carpometacarpal joint of the thumb in osteoarthritis. Ann Rheum Dis 2004;63:1260-3.

4. Stahl S, Karsh-Zafrir I, Ratzon N, et al. Comparison of intraarticular injection of depot corticosteroid and hyaluronic acid for treatment of degenerative trapeziometacarpal joints. J Clin Rheumatol 2005;11:299-302.

5. Fuchs S, Mönikes R, Wohlmeiner A, et al. Intra-articular hyaluronic acid compared with corticoid injections for the treatment of rhizarthrosis. Osteoarthritis Cartilage 2006;14:82-8.

6. Heyworth BE, Lee JH, Kim PD, et al. Hylan versus corticosteroid versus placebo for treatment of basal joint arthritis: a prospective, randomized, double-blinded clinical trial. J Hand Surg Am 2008;33:40-8.

7. Monfort J, Rotés-Sala D, Segalés N, et al. Comparative efficacy of intra-articular hyaluronic acid and corticoid injections in osteoarthritis of the first carpometacarpal joint: results of a 6-month single-masked randomized study. Joint Bone Spine 2015;82:116-21.

8. Roux C, Fontas E, Breuil V, et al. Injection of intra-articular sodium hyaluronidate (Sinovial) into the carpometacarpal joint of the thumb (CMC1) in osteoarthritis. A prospective evaluation of efficacy. Joint Bone Spine 2007;74:368-72.

9. Bahadır C, Onal B, Dayan VY, et al. Comparison of therapeutic effects of sodium hyaluronate and corticosteroid injections on trapeziometacarpal joint osteoarthritis. Clin Rheumatol 2009;28:529-33.

10. Mandl LA, et al. A randomized controlled trial of hylan G-F 20 for the treatment of carpometacarpal osteoarthritis. Arthritis Rheum 2012;64:S475-6.

11. Ozturk C, Atamaz F, Hepguler S, et al. The safety and efficacy of intraarticular hyaluronan with/without corticosteroid in knee osteoarthritis: 1-year, single-blind, randomized study. Rheumatol Int 2006;26:314-9.

12. Petrella RJ, Emans PJ, Alleyne J, et al. Safety and performance of hydros and hydros-TA for knee osteoarthritis: a prospective, multicenter, randomized, double-blind feasibility trial. BMC Musculoskelet Disord 2015;16:57.

13. Siengdee $P$, Radeerom $T$, Kuanoon $S$, et al. Effects of corticosteroids and their combinations with hyaluronanon on the biochemical properties of porcine cartilage explants. BMC Vet Res 2015;11:298.

14. Kroon FP, Rubio R, Schoones JW, et al. Intra-articular therapies in the treatment of hand osteoarthritis: a systematic literature review. Drugs Aging 2016;33:119-33.

15. Trellu S, Dadoun S, Berenbaum F, et al. Intra-articular injections in thumb osteoarthritis: a systematic review and meta-analysis of randomized controlled trials. Joint Bone Spine 2015;82:315-9.

16. Chan AW, Tetzlaff JM, Gøtzsche PC, et al. SPIRIT 2013 explanation and elaboration: guidance for protocols of clinical trials. BMJ 2013;346:e7586.

17. Di Sante L, Cacchio A, Scettri P, et al. Ultrasound-guided procedure for the treatment of trapeziometacarpal osteoarthritis. Clin Rheumatol 2011;30:1195-200.

18. Dreiser RL, et al. Ibuprofen $800 \mathrm{mg}$ for the treatment of osteoarthritis of the interphalangeal joints of the hand on trapezo-metacarpal joint. Rev Rhum 1993;60:719-24.

19. Poiraudeau S, Chevalier X, Conrozier T, et al. Reliability, validity, and sensitivity to change of the Cochin hand functional disability scale in hand osteoarthritis. Osteoarthritis Cartilage 2001:9:570-7.

20. Angst F, Drerup S, Werle S, et al. Prediction of grip and key pinch strength in 978 healthy subjects. BMC Musculoskelet Disord 2010;11:94

21. Dougados M. Principes généraux de l'évaluation des affections rhumatismales: évaluation symptomatique des affections rhumatismales in la mesure: méthodes d'évaluation des affections rhumatismales. Éd Expansion Scientifique Publications 1997;7-20:62. 